Revue internationale P.M.E.

Économie et gestion de la petite et moyenne entreprise

Revure

internationale

PME

\title{
Le rôle des petites entreprises dans la transformation vers l'économie de marché dans l'ex-Yougoslavie
}

\section{Berislav Sefer}

Volume 5, numéro 1, 1992

URI : https://id.erudit.org/iderudit/1008134ar

DOI : https://doi.org/10.7202/1008134ar

Aller au sommaire du numéro

Éditeur(s)

Presses de l’Université du Québec

ISSN

0776-5436 (imprimé)

1918-9699 (numérique)

Découvrir la revue

Citer cette note

Sefer, B. (1992). Le rôle des petites entreprises dans la transformation vers l'économie de marché dans l'ex-Yougoslavie. Revue internationale P.M.E., 5(1), 77-92. https://doi.org/10.7202/1008134ar

\section{Résumé de l'article}

Cet article porte sur le rôle des petites entreprises dans la transformation de l'économie de l'ex-Yougoslavie vers une économie de marché. Malgré les conditions politiques actuelles où l'économie est désintégrée et sensible- ment dévastée par la guerre et sans égard aux futures solutions politiques, le rôle des PME dans la transformation de l'économie, malgré tout devrait passablement se ressembler pour toutes les républiques qui formaient la Yougoslavie.

L'auteur montre que l'importance du développement des petites entreprises dans la transformation du système politique et de celui du marché est non seulement très grande, mais qu'en réalité, la transformation démocratique et économique dépendra en grande mesure du développement des petites entreprises. Quoique les possibilités de développement de ces entreprises soient nombreuses (restructuration agraire, main-d'œuvre de qualité, conditions économiques générales favorables, présence de capitaux, capacités énergétiques), on trouve aussi beaucoup de contraintes à ce développement: l'ancienne mentalité, un esprit d'entreprise inégal selon les régions, un système de communication désuet et, bien sûr, la guerre actuelle qui rend les perspectives d'avenir tellement incertaines.
Ce document est protégé par la loi sur le droit d'auteur. L'utilisation des services d’Érudit (y compris la reproduction) est assujettie à sa politique d'utilisation que vous pouvez consulter en ligne.

https://apropos.erudit.org/fr/usagers/politique-dutilisation/ 


\title{
Le rôle des petites entreprises dans la transformation vers l'économie de marché dans l'ex-Yougoslavie
}

\author{
Dr Berislav SEFER \\ Université pour la Paix des Nations Unies*
}

\begin{abstract}
RÉSUMÉ
Cet article porte sur le rôle des petites entreprises dans la transformation de l'économie de l'ex-Yougoslavie vers une économie de marché. Malgré les conditions politiques actuelles où l'économie est désintégrée et sensiblement dévastée par la guerre et sans égard aux futures solutions politiques, le rôle des PME dans la transformation de l'économie, malgré tout devrait passablement se ressembler pour toutes les républiques qui formaient la Yougoslavie.

L'auteur montre que l'importance du développement des petites entreprises dans la transformation du système politique et de celui du marché est non seulement très grande, mais qu'en réalité, la transformation démocratique et économique dépendra en grande mesure du développement des petites entreprises. Quoique les possibilités de développement de ces entreprises soient nombreuses (restructuration agraire, main-d'œuvre de qualité, conditions économiques générales favorables, présence de capitaux, capacités énergétiques), on trouve aussi beaucoup de contraintes à ce développement: l'ancienne mentalité, un esprit d'entreprise inégal selon les régions, un système de communication désuet et, bien sûr, la guerre actuelle qui rend les perspectives d'avenir tellement incertaines.
\end{abstract}

* Berislav Sefer Ph.D., diplômé de la faculté d'Économique de l'Université de Zagreb, maintenant à la retraite, est conseiller spécial du Centre européen pour la paix et le développement à l'Université pour la Paix des Nations Unies. Domaines de spécialisation : ressources humaines en économie, les niveaux de vie, les petites entreprises et la politique sociale et le marché. Il a publié plus de 20 volumes et quelques 200 articles scientifiques, en Yougoslavie et à travers le monde.

Adresse : Centre Européen pour la Paix et le Développement, Kneza Mihaila 7/II, 11000 Beograd, Yougoslavie. 


\begin{abstract}
This article deals with the role of small business in the economic transformation of the former Yugoslavia toward a market economy. Despite the present political conditions in which the economy is disintegrated and substantially devastated by the war and without considering future political solutions, the role of SMBs in economic transformation should in spite of all come together somewhat in all the republics which make up Yugoslavia.

The author shows that the importance of the development of small business in the transformation of the political system and that of the market is not only crucial, but in reality the democratic and political transformation depends largely on the development of small business. However numerous the possibilities of small business development (agrarian restructurization, competent manpower, availability of capital, energy capacity) one also finds many constraints to this developpement: an antiquated mentality, an entrepreneurial spirit which is uneven throughout the regions, an outdated communications system and of course the present war which makes future perspectives very uncertain.
\end{abstract}

\title{
RESUMEN
}

Este artículo toca sobre el rol de PME dentro de la transformacion de la económia de la ex-Yugoslavia hacia una económia de mercado. A pesar de las condiciones políticas actuales donde la económia es desintegrada y sensiblemente devastada por la guerra y sin consideracion a las futuras soluciones políticas, el rol de las PME dentro de la transformacion de la económia devería a pesar de todo parecerse practicamente por todas las republicas que forma la Yugoslavia.

El autor muestra que el desarrollo de las PME dentro de la transformacion del sistema político y del mercado no es solamente muy grande pero en realidad, la transformacion democratica y económica dependera en una gran medida del desarrollo de las PE. Sin importar las posibilades de desarrollo de PE sean numerossas (reestructuracion agragia, mano de obra de calidad, condiciones económicas generales favorables, presencia de capital, capacidades energeticas) uno encuentre tambien mucho de limitaciones a este desarrollo: la antigua mentalidad, un espiritu de empresa inigual segun las regiones, un sistema de comunicacion obsoleto y naturalmente la guerra actual que hace de las perspectivas del avenir verdadermente inciertas.

\section{Introduction}

Cet article porte sur le rôle des petites entreprises dans la transformation de l'économie de l'ex-Yougoslavie vers l'économie de marché, pays en pleine désintégration. Le modèle de l'économie yougoslave s'est développé durant à peu près soixante-dix ans. Dans l'entre-deux-guerres, les conditions d'une économie de marché furent retardées par les besoins d'une économie de survie. 
Après la Deuxième Guerre mondiale, cette économie s'est faite sous les conditions de propriété sociale et d'autogestion. Dans ce contexte, la situation économique yougoslave actuelle résulte des conditions sociales dans lesquelles elle s'est développée, peu importe les républiques. C'est dans ce sens que le rôle et la situation des petites entreprises en Yougoslavie peuvent être analysés quelles que soient les républiques de l'ex-Yougoslavie.

Dans les conditions politiques actuelles, on ne peut parler de l'économie yougoslave que conditionnellement, car on ne sait pas ce qu'elle sera dans l'avenir. Mais à cause des dizaines d'années de développement commun, les caractéristiques des économies des républiques sont communes. Lorsque la guerre actuelle sera terminée, lorsque les esprits seront devenus sereins et que les passions nationalistes se seront calmées, il sera dans l'intérêt de tous, voire nécessaire, que toutes les républiques coopèrent sur un large plan économique pour créer des conditions optimales de développement pour leurs économies.

Dans les conditions actuelles, on ne peut parler de l'économie yougoslave qu'en termes d'espace géographique. L'économie est désintégrée et sensiblement dévastée par la guerre. Elle est plus loin de l'économie de marché qu'elle ne l'a été il y a quelques années. Mais, sans égard aux futures solutions politiques, la « reconstruction au lendemain de la guerre » et la transformation du marché auront, pour toutes les républiques qui formaient la Yougoslavie jusqu'à présent, des caractéristiques communes. Le rôle des petites et moyennes entreprises dans la transformation de cette économie vers l'économie de marché devrait donc passablement se ressembler.

\section{Le petit nombre de PME dans l'économie de l'ex-Yougoslavie : un trou noir}

Dans les pays développés à l'économie de marché, on sait que le rôle des petites entreprises est grand. L'expérience de ces pays est connue, même dans les pays possédant un autre type d'économie. Ces entreprises contribuent à la croissance, à l'accroissement du revenu et de l'emploi et à l'insertion de l'économie dans le marché mondial. Après les années 70 , ce rôle des PME dans le développement économique des pays développés à économie de marché s'est accru. C'est le résultat des changements structurels dans ces économies. Une nouvelle demande a exigé un plus large assortiment que celui que pouvait donner la production en série; les nouvelles technologies (surtout microélectronique et de communication) ont favorisé les petites unités de production. Une grande capacité des petites entreprises pour l'innovation et une forte tendance vers les services 
personnels se sont ajoutées. Ainsi, c'est dans une période de restructuration des économies de marché et lorsque leur développement est devenu plus dynamique que les petites entreprises se sont révélées un facteur essentiel pour atténuer ou limiter les effets sociaux de cette restructuration.

Cette expérience dans le développement des petites entreprises des pays à économie de marché n'a pas servi aux pays à économie centralisée, comme la Yougoslavie. Le système économique yougoslave était basé sur le monopole de la propriété d'État et sur la gestion administrative et centralisée de l'économie. Ce système était caractéristique jusqu'à la moitié des années 50, alors que la propriété d'État s'est transformée en propriété sociale et qu'elle fut donnée aux collectifs de travail qui la gérèrent désormais. C'est ainsi qu'on a introduit l'autogestion, soit un système économique qui repose sur des rapports de nonpropriété. Le marché n'agit que dans le domaine des marchandises, mais non dans celui du travail et du capital. C'est pourquoi la coordination du développement dans de telles conditions se fait bureaucratiquement et administrativement et non par le marché.

Le système économique yougoslave basé sur la propriété sociale et sur l'autogestion présentait un système économique plus souple par rapport à celui des pays à économie planifiée centralement. Cependant, comme ce système se basait sur des rapports de non-propriété et sur un marché étiolé, le développement économique est resté grandement décentralisé, même s'il passait par les républiques et par les organes locaux. Il s'agissait donc d'un système économique spécifique de non-marché géré et coordonné administrativement et bureaucratiquement d'abord par les autorités républicaines.

C'est sur ces bases économiques que le rapport des autorités face aux petites entreprises s'est fait. En Yougoslavie, créer des grandes entreprises était presque devenu une obsession, car on croyait que ces grands ensembles étaient économiquement les plus efficaces, mais aussi les plus propices à la gestion bureaucratico-administrative et au contrôle du marché, contrairement aux petites entreprises. Stimulées par la politique officielle, ces grosses entreprises ont continué à grandir, surtout par différentes formes d'intégrations, souvent tout à fait efficaces du point de vue économique. Dans une telle situation, aucune nouvelle entreprise ne se formait. S'il y a eu des tentatives pour stimuler le développement de petites entreprises en tant qu'élément essentiel de la structure économique, ce fut pratiquement sans aucun résultat, malgré plusieurs programmes au niveau de l'État.

Une autre raison pour laquelle le développement des petites entreprises a été négligé réside dans les fondements idéologiques du système économique, lequel est basé sur des rapports de non-propriété, sur la gestion administrative et sur la limitation du marché. Les petites entreprises sont des entreprises de 
« marketing »; elles agissent directement sous l'influence de l'impulsion du marché ; elles n'ont donc pas pu s'insérer ni dans le système économique basé sur la non-propriété, ni dans la coordination administrative qui remplaçait celle du marché.

Le secteur privé était limité. Il existait dans l'agriculture, l'artisanat, le tourisme et dans différents services, mais aussi dans des petits ateliers de production. Jusqu'à la moitié des années 80 , l'embauche de la main-d'œuvre salariée par les entreprises privées était traitée comme des exceptions ; en pratique, on tolérait jusqu'à une dizaine de salariés. Mais le secteur privé était contraint par d'autres mesures.

Les charges fiscales et les contributions pour la sécurité sociale, la santé, etc., étaient toujours plus élevées pour le secteur privé que pour le secteur collectif ; la politique monétaire, de crédit et de devises, favorisait le secteur collectif et limitait le privé. Dans ces conditions, le secteur privé ne pouvait qu'être en marge du développement et de la politique économique ; de sorte qu'il n'y a pas eu de développement de petites entreprises.

De plus, le système économique a défendu l'autarcie de l'économie yougoslave face au marché international. Lorsque la direction administrative et bureaucratique de l'économie eut quitté le centre de la fédération pour passer aux républiques, l'autarcie intérieure ne put que se renforcer. Ceci a restreint encore davantage l'échange de biens. L'influence administrative sur le marché est devenue de plus en plus grande, soit indirectement à travers les instruments de la politique économique, soit directement à travers les interventions sur les prix.

C'est ainsi que le marché n'a pu agir sélectivement. L'économie sociale était hautement protégée, toutes les entreprises collectives se maintenaient même si elles étaient peu efficaces. Les entreprises privées (pour autant qu'elles se développaient) ne pouvaient pas progresser même si elles étaient efficaces. Cette marche des affaires était donc davantage basée sur une faible efficacité et une faible compétitivité des entreprises sociales que sur la concurrence économique du secteur privé. Par son action ainsi dénaturée, le marché n'a pu agir, pas plus sur les petites entreprises manufacturières que sur les petits commerces privés, pour ne retenir que les plus efficaces.

Le fait que l'offre soit toujours inférieure à la demande avait aussi un effet défavorable sur le développement des petites entreprises. Sur le marché des biens, la demande se développait alors que le consommateur demeurait en situation d'infériorité. Tout se vendait même si c'était des marchandises médiocres. Par conséquent, l'influence de la demande a plutôt nui à la diversification de l'offre et à l'amélioration de la qualité, deux facteurs essentiels pour le développement des petites entreprises dans les économies de marché. 
Dans ces conditions, les petites entreprises privées légales ont été remplacées en partie par l'offre de marchandises et de services sur le marché noir. Le marché noir existe dans tous les pays à économie de marché. Mais dans les conditions de monopole des entreprises sociales, les faibles revenus de ceux qui y travaillent et le peu de motivation qu'ils y trouvent en ont incité plusieurs à avoir un travail complémentaire. Ceci a donné lieu à de multiples effets défavorables. Bon nombre de ceux qui travaillaient dans le secteur collectif passaient leur temps à travailler pour leur propre compte, ou encore travaillaient mal, gardant leur énergie pour le travail complémentaire privé. Plusieurs n'étaient intéressés qu'à fournir le travail minimum nécessaire pour conserver leur emploi et être admissibles à la sécurité sociale et la sécurité-retraite. Mais cela allait plus loin encore : pour ce travail complémentaire, on utilisait les outils et parfois même la matière première de l'entreprise collective où on travaillait. D'une part, ce travail complémentaire dans ces circonstances sociales signifiait donc un mauvais rendement dans l'entreprise sociale et menait souvent à des vols d'outils et de matériel. D'autre part, la qualité de la marchandise et des services provenant de travail complémentaire offerte aux consommateurs était également médiocre et la qualité, nulle. Dans ces circonstances, le marché noir ne pouvait pas remplacer le sous-développement des petites entreprises.

\section{DiAgramme 1}

Distribution des entreprises collectives et privées d'après le nombre de personnes employées dans l'industrie manufacturière en Yougoslavie et en Suède

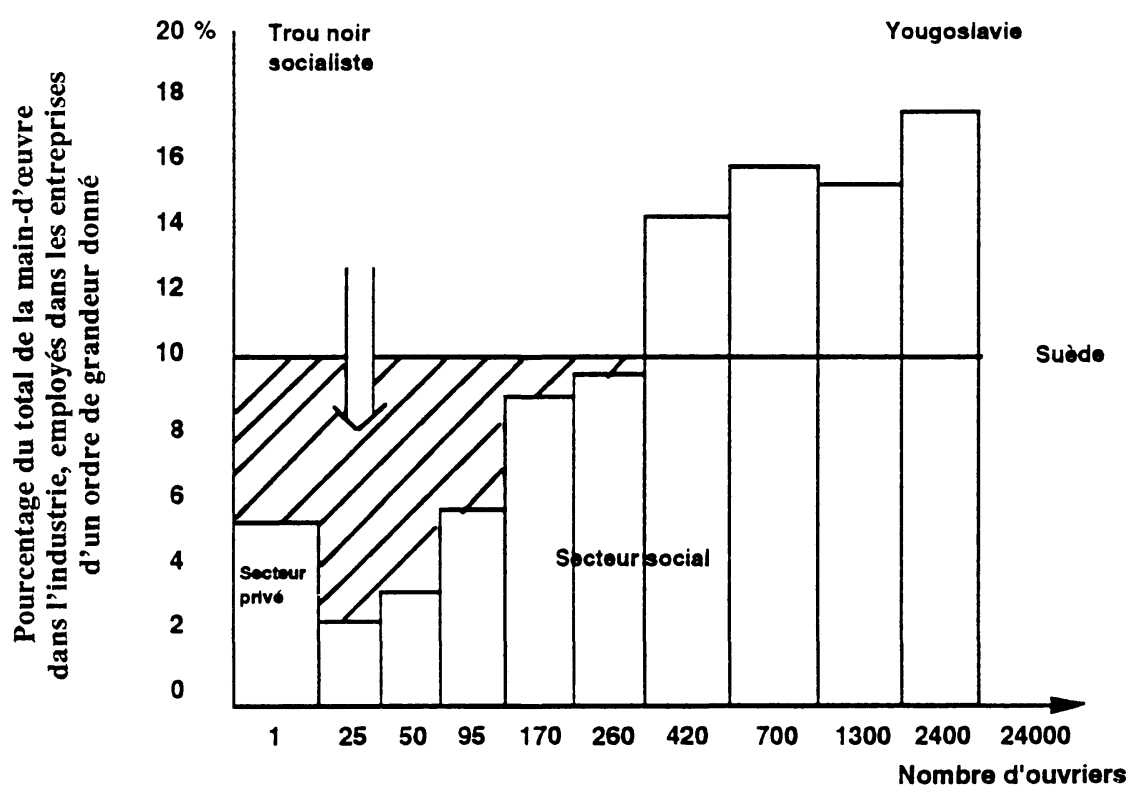

Revue internationale P.M.E., vol. 5, $\mathrm{n}^{\circ} 1,1992$ 
Pour cette raison, on peut dire que l'économie yougoslave (ce qui est valable pour toutes les républiques) est structurellement déséquilibrée, tant dans le sens d'une pénurie en petites entreprises que dans celui de la faiblesse généralisée au plan de l'efficacité et de la compétitivité. Le petit nombre de petites entreprises présente donc un « trou noir » dans la structure économique. Le diagramme qui suit basé sur la comparaison de la structure de l'industrie yougoslave et de celle de la Suède fait bien ressortir ce déséquilibre.

Le diagramme de la page précédente montre bien que les petites entreprises n'étaient pratiquement pas présentes vers le milieu des années 80 , (donc avant le début de la réforme économique radicale) dans la structure économique de la Yougoslavie. Mais même lorsqu'on parle des entreprises privées, il s'agit en réalité de micro entreprises (plutôt des petits commerces, que des petites entreprises manufacturières).

La réforme économique radicale annoncée en 1989 a commencé à être mise en œuvre en 1990. La réforme repose sur :

- le développement du marché intégral (travail, services et capitaux);

- le développement d'une économie ouverte et son intégration dans l'économie mondiale, surtout européenne ;

- la prépondérance des droits définis par la loi, et promulgués à la suite d'une procédure démocratique (dans un parlement multipartis) sur les normes politiques et idéologiques ;

- l'édification d'une société et d'un système partant de l'individu, de ses capacités, des préférences des consommateurs, des droits et des libertés personnelles.

Les premières lois concernant la mise en œuvre d'une réforme économique aussi fondamentale ont été votées à la fin de 1989. Elles donnaient l'espoir de voir cette transformation vers l'économie de marché se faire avec relativement peu de pertes et surtout de faibles conséquences sociales. Le développement du secteur privé avec la création d'entreprises privées et la privatisation des entreprises publiques devait occupé une place très importante. Mais l'accent mis sur le développement des petites entreprises était encore plus manifeste avec la création d'une Agence fédérale des PME ayant des succursales dans toutes les républiques. Mais dès le début, cette réforme a été accueillie de façon différente de la part des autorités républicaines. En principe, toutes les républiques ont accepté le concept de la réforme économique, mais pas nécessairement les mesures pratiques et significatives pour son application. La résistance aux nouvelles règles apportées par ces nouvelles lois furent souvent très forte. 
Dans certaines républiques, les lois fédérales ne furent pas appliquées. Cette résistance a ralenti dès la deuxième moitié de 1990 l'application de la réforme économique et, vers la fin, tout était bloqué. Les conflits politiques et nationaux se sont transformés en conflits armés qui ont assujetti complètement la gestion économique à la situation de guerre. Dans une telle situation, il est difficile d'écrire quoi que ce soit qui demeure d'actualité, car la situation change constamment. Économiquement et socialement, la situation économique est de plus en plus mauvaise et s'éloigne de plus en plus de l'économie de marché, bien qu'aucune des autorités républicaines ne mette en cause l'intégrité de la réforme. À cause de cela, il est difficile de faire toute estimation concernant l'aptitude réelle du pays à transformer son système en économie du marché. Tout ce qui sera subséquemment exposé constitue donc un ensemble de faits et d'estimations quant au rôle des petites entreprises dans la transformation économique et dans la définition des principes de marché en Yougoslavie. La réalité montrera comment cette transformation pourra se développer après la guerre.

\section{Les petites entreprises dans la transformation de l'économie yougoslave}

La transformation vers l'économie de marché de l'ex-Yougoslavie a pour objectif :

- la revitalisation de l'économie et l'augmentation de l'efficacité économique,

- la création de conditions pour que chacun puisse s'assurer le bien-être et la sécurité par son travail, sa connaissance et sa compétence, et

- la création des conditions économiques pour un système politique démocratique.

L'objectif final est d'élever le niveau et la qualité des conditions de vie dans le sens le plus large de ce mot.

Dans cette optique, on peut affirmer que les petites entreprises possèdent des caractéristiques et des qualités économiques, technologiques et sociales pouvant jouer un très grand rôle dans la restructuration de l'économie yougoslave. Mais, dans le développement actuel, elles n'ont pas encore été exploitées comme facteur de croissance économique. La partie, en pourcentage, des petites entreprises dans le produit national l'indique clairement. De même, on n'a pas recouru aux possibilités que les petites entreprises offrent pour un accroissement sain de l'emploi. Les petites entreprises travaillent de façon intensive, car on exige d'elles du savoir et de la dextérité. Bien que la structure 
qualitative de la population se soit sensiblement améliorée, le savoir n'a pas, jusqu'à présent, été exploité comme facteur de développement. La motivation pour un travail efficace dans les grandes entreprises collectives demeure faible. Dans les petites entreprises privées, le savoir, la dextérité et la motivation sont à la base de l'efficacité du travail. Dans l'économie yougoslave, le développement des petites entreprises demeure pour ainsi dire un espace économique inoccupé ; leur développement favoriserait le processus de revitalisation des entreprises qui, ainsi, profiteraient de ressources économiques et humaines mal utilisées jusqu'à présent.

On peut illustrer ceci par l'exemple des ressources énergétiques, qui sont importées pour la plus grande partie. Dans des conditions de déficit de la balance de paiement et de l'amortissement des dettes à l'étranger, l'importation de l'énergie constitue une grande charge qui, souvent, par manque de devises, entraîne des ruptures dans l'approvisionnement énergétique, surtout en pétrole. Les petites entreprises n'étant pas d'intenses consommatrices d'énergie, leur développement devrait diminuer cette dépendance.

De même, les investissements par poste de travail dans les petites entreprises sont 2 à 2,5 fois moins importants que dans les grandes entreprises. Comme l'économie de l'ex-Yougoslavie est très en retard (encore plus maintenant, à cause de la guerre) elle n'a pratiquement plus d'épargne " nationale ». Dans ces conditions, il est important de favoriser le développement de petites entreprises qui réussissent à récupérer l'épargne individuelle ou familiale, à obtenir du capital en tant que sociétés anonymes, ou à emprunter sur leurs actifs. Ce sont, dans les conditions actuelles les seules ressources réelles de capitaux. Avec le temps, ce capital pourra être concentré pour de plus grandes exploitations économiques et technologiques.

La technologie dans l'économie de l'ex-Yougoslavie souffre d'un retard sensible dû à la stagnation, voire même à une importante chute de l'activité économique. Cependant, l'économie n'a plus de capitaux qui lui permettraient de promouvoir rapidement sa technologie. Sans privatisation et sans importante participation du capital étranger, la transformation technologique n'est pas possible. Mais les expériences dans le monde ont montré que les petites entreprises s'adaptaient facilement aux nouvelles technologies. L'expérience yougoslave, bien qu'au total la petite entreprise soit sous-développée, a montré qu'elle était ouverte aux innovations et s'adaptait facilement aux changements technologiques. Dans cette transformation, les petites entreprises peuvent et doivent jouer un rôle très important.

Dans la période de transformation du marché, l'économie yougoslave doit faire face à des problèmes sociaux exceptionnels. La situation sociale est très mauvaise et ne fait que s'aggraver. Le nombre de chômeurs dépasse 1,6 millions 
de personnes, auxquelles il faut ajouter au moins 1500000 personnes provenant du chômage déguisé dans les entreprises. La privatisation des entreprises publiques ne peut se faire sans transférer l'excédent de personnes employées dans d'autres activités économiques. Le développement des petites entreprises est le meilleur moyen pour augmenter l'embauche ; toutes les autres semblent peu efficaces sinon inexistantes. Le développement des petites entreprises est non seulement important pour limiter le chômage, mais il constitue un facteur essentiel qui accélérera le processus de privatisation des entreprises collectives.

Sur le plan social, le développement des petites entreprises peut avoir plusieurs effets favorables. Tout d'abord, il suscite le développement d'autres productions économiques, ce qui permet à la population de satisfaire ses besoins. Cet effet est important, spécialement en ce moment, alors que le niveau de vie se détériore sensiblement. Il convient de mentionner aussi que le développement des petites entreprises est un processus décentralisé qui réduira la pression de l'exode rural vers les villes, alors que c'est justement là qu'il y a le plus de problèmes sociaux.

On peut penser aussi que l'atmosphère de travail et les rapports interpersonnels sont plus agréables dans les petites entreprises que dans les grandes. Les relations humaines se sont détériorées surtout dans les entreprises collectives depuis la période d'autogestion et du système de non-propriété. La répartition du revenu dans les entreprises était plus ou moins égalitaire et n'était pas liée aux résultats du travail des individus. Voyons cela de façon théorique au diagramme 2.

DiAgramme 2

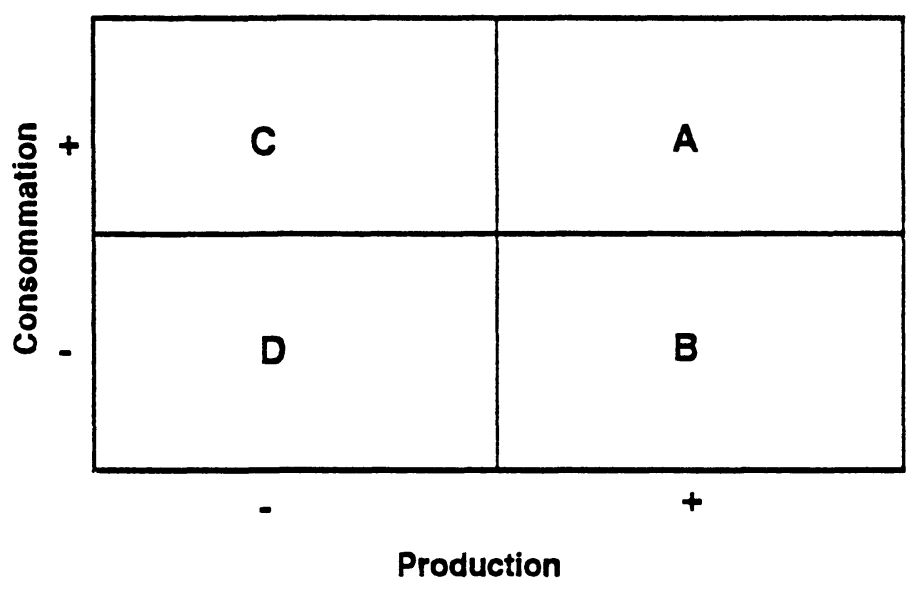

Revue internationale P.M.E., vol. 5, n 1, 1992 
Ce diagramme est divisé en 4 quadrants. Chaque quadrant représente les travailleurs du secteur collectif, d'après leur participation à la production et leur revenu pour consommation. Ainsi, on peut voir que le groupe $C$ contribue peu à la production, mais reçoit beaucoup pour consommer ; le groupe $\mathrm{B}$ donne beaucoup à la production, mais il reçoit très peu pour la consommation, etc. Bien que ce diagramme ne puisse être quantitativement évalué, il donne une image réelle de la répartition de la consommation par rapport à la contribution de travail dans les entreprises collectives. Ainsi, nous savons par la pratique que le groupe $\mathrm{A}$ est très petit. Le groupe $\mathrm{C}$ est bien plus grand, il produit peu, mais reçoit beaucoup pour la consommation. C'est le groupe que le système a privilégié. Le groupe $\mathrm{B}$ ne reçoit pas l'équivalent de sa contribution à la production. Enfin, le groupe D qui contribue peu et reçoit peu est le plus grand, numériquement. C'est le groupe dominant et, avec le groupe $C$ qui est privilégié, ils sont en somme contre la transformation de la propriété et le passage à l'économie de marché.

Dans les entreprises privées, surtout dans les petites, le modèle de la distribution est basé sur le bénéfice qui correspond à la contribution dans la production. Ceci contribue à améliorer l'atmosphère et les relations interpersonnelles au travail. Finalement, ceci est à l'origine d'une très grande motivation au travail.

Étant donné le niveau de développement, l'environnement naturel est fort dégradé. Même si l'on veut améliorer la situation écologique, les petites entreprises ont d'importants avantages. Elles dégradent moins le milieu naturel, elles ne requièrent pas une concentration urbaine ; de ce fait même, elles réduisent les conséquences écologiques défavorables d'une telle concentration.

Parmi les objectifs de la transformation de l'économie en économie de marché, il convient de mentionner la création de conditions économiques pour soutenir un système politique démocratique. Dans ce processus, les petites entreprises sont appelées à jouer un rôle important. C'est là que se développe et qu'est valorisé l'esprit d'entreprise. Les petites entreprises créent des conditions économiques nécessaires à l'indépendance économique des particuliers par rapport à celle de l'État. Il est certain que ceci est une des conditions essentielles pour la démocratisation sociale.

Le développement des petites entreprises contribue à la transformation vers l'économie de marché et joue ainsi non seulement un rôle économique mais aussi social et politique. C'est pour cette raison que leur développement ne doit pas se faire en marge de la transformation du marché, mais doit être un des éléments majeurs de cette transformation. 


\section{Possibilités et limites du développement des petites entreprises dans l'économie de l'ex-Yougoslavie}

L'importance du développement des petites entreprises dans la transformation du système politique et de celui du marché, tant sur le plan économique que social, est très grande. En réalité, la transformation démocratique et économique dépendra en grande mesure du développement des petites entreprises.

Les possibilités de développement des petites entreprises sont nombreuses :

- Il convient tout d'abord de souligner les conditions au plan de l'exploitation agricole, une politique agraire qui reposerait sur la restructuration de la propriété agricole pourrait encourager les petites exploitations et les fermes, en plus des exploitations familiales. On verrait alors les terres agricoles être exploitées de façon à augmenter rapidement la production de nourriture et de matières premières agricoles. De plus, les conditions naturelles permettent de développer le tourisme (mer, montagnes, lacs, situation géographique, etc.). Avant la guerre civile, le tourisme était déjà développé avec la participation importante du secteur privé. Mais les possibilités du secteur privé, pour l'expansion du tourisme, sont loin d'être tout à fait exploitées.

- Il existe une abondante main-d'œuvre qualifićc, instruite et bien formée. Dans le secteur collectif, la moyenne du nombre d'année d'études des travailleurs est de 11 ans, et celle des chômeurs est même quelque peu plus élevée : 11,4 années. Cependant, à cause du type du système économique et du système d'enseignement, la main-d'œuvre a été formée pour une organisation de travail hiérarchique. La formation reçue est donc lacunaire en ce qui a trait à l'esprit d'entreprise, au management et, en général, à l'organisation du travail tel que le demande un marché développé. Cette situation créée par l'ancien système pourrait toutefois être corrigée par un complément de formation et de pratique.

- Les conditions économiques générales facilitent le développement des petites entreprises. Tout d'abord, le capital se trouve là où sont les épargnes des citoyens. Ces épargnes s'élèvent à environ 15 milliards de dollars. Il s'agit là d'épargnes pour lesquelles les banques ont imposé de fortes restrictions quant à leur utilisation étant donné que, par leur intermédiaire, il a permis divers investissements. Mais après la débâcle du système économique et financier, les épargneurs ont, pour le 
moment, peu de possibilités pour l'utiliser. Cependant, il y a d'importantes épargnes hors du système bancaire. Il est difficile d'en donner une appréciation exacte, mais il s'agit vraisemblablement d'un montant de plus de 5 milliards de dollars. Les ouvriers migrants ont déposé d'importantes sommes dans les banques à l'étranger, qu'ils investiraient certainement dans le pays si la guerre finissait et si une stabilité politique était créée. Dans ces conditions-là, on pourrait s'attendre aussi à une entrée des capitaux étrangers.

Mais outre sur les ressources financières, il faut attirer l'attention sur l'énorme capital non exploité que représentent les bâtiments, bureaux et équipement dans les entreprises collectives. En 1989, on avait évalué leur valeur à 50 milliards de dollars. Cette somme est égale au produit national brut d'une année en Yougoslavie. Il est vrai que parmi ces équipements non utilisés, certains sont vétustes et non utilisables. Mais il y a tout cet espace utilisable qui, avec la privatisation, serait fonctionnel pour les affaires. Il faut donc compter avec la mise en service de cet énorme capital non exploité pour le développement des petites entreprises.

Il faut ajouter que l'infrastructure est, en général, satisfaisante pour le développement des petites entreprises (capacités énergétiques, voies de transport, etc.). Si elle était mieux utilisée, elle constituerait une grande incitation pour la revitalisation de l'économie et sa transformation au point de vue de la propriété. Naturellement, toutes ces estimations sont sujettes à de nouvelles analyses liées aux destructions causées par la guerre.

Par contre, il existe plusieurs obstacles au développement des petites entreprises. Nous nous bornerons à n'en citer que quelques-unes.

- L'influence très forte de la mentalité qui a longtemps prévalu dans l'économie, mentalité tant sociale qu'individuelle selon laquelle la source du développement se trouve dans l'État et les autorités au pouvoir et non dans l'initiative et la motivation des gens.

- Un esprit d'entreprise, une créativité et une aptitude à prendre des risques très faibles. On trouve encore un climat défavorable à l'esprit d'entreprise, contre le risque, bref un climat de médiocrité.

- La nouvelle politique agraire qui repose sur le développement des petites fermes rencontre beaucoup de difficultés à cause des rapports économiques et sociaux complexes dans l'agriculture.

- Les grandes entreprises collectives sont en crise. Elles n'ont plus de capitaux, elles ne sont pas en mesure de stimuler le développement des petites entreprises ; très souvent, elles ne sont même pas intéressées à 
le faire, car elles ont peur de la concurrence qu'elles pourraient leur faire, soulignant leur inefficacité, si on passe réellement à l'économie de marché.

- L'attitude envers l'esprit d'entreprise est différente d'une région à l'autre. Il y a des régions où cette résistance est encore très forte. Elle est particulièrement forte là où l'on s'oppose à la privatisation et au marché.

- Dans l'infrastructure, le système de communication a du retard; ce système est pourtant essentiel pour soutenir l'efficacité des petites entreprises (téléphone, télex, télécopieur, etc.).

- Le système économique est loin d'être orienté vers l'esprit de marché ; la politique économique change tout le temps (en ce moment elle est soumise à l'État de guerre), de sorte que l'on ne peut planifier des changements économiques draconiens même pour un terme très court. Le risque est exceptionnellement élevé.

- La guerre et l'instabilité politique ont détruit toute confiance dans l'avenir.

Autant les possibilités de développement des petites entreprises sont grandes, autant les limites le sont. Avec la fin de la guerre et le retour à la stabilité politique, l'importance de ces limites se réduira, de sorte que les petites entreprises pourront jouer un rôle très important dans la transformation économique dans le sens de la propriété et du marché.

\section{Perspectives}

Il n'y a rien de plus ingrat que de parler des perspectives du développement des petites entreprises dans l'ex-Yougoslavie. Comme le sort de tout le « pays » est incertain, la transformation de la propriété et du marché et, par ce fait même, le développement des petites entreprises sont tout aussi problématiques.

Malgré le fait que la situation économique, déjà très mauvaise, ait tendance à se détériorer rapidement, surtout à cause de l'état de guerre, le nombre de firmes privées (surtout des petites entreprises) a augmenté jusqu'en 1990 de $70 \%$ environ, comparativement à l'année 1984, avec une légère tendance à la hausse dans la première moitié de 1991. Dans les petites entreprises privées, le nombre de salariés s'est accru de $135 \%$; la moyenne par firme a augmenté de $13 \%$. Mais il s'agit là de micro-entreprises où le nombre de salariés moyen est 
inférieur à 2 personnes. Il est intéressant de souligner que le plus grand nombre de ces micro-entreprises ont été créées en Serbie (environ $35 \%$ ), ensuite en Croatie $(23 \%)$ et Slovénie $(15 \%)$. Même dans des conditions très défavorables, le processus de création des petites entreprises se poursuit, bien que moins rapidement qu'en 1990. Étant donné que le PIB provenant du secteur collectif a été en 1990 de $35 \%$ au moins, alors que le PIB total a atteint au moins $200 \%$, la participation actuelle des petites entreprises (en majorité privées) dans le PIB du pays est passé à $11 \%$ environ, comparativement à $6 \%$ en 1988.

Dans toutes les républiques de l'ex-Yougoslavie, on a fort simplifié les conditions légales pour la création des entreprises privées ; il n'y a pratiquement plus de problèmes graves, ni de contraintes. Les besoins de bureaux et de locaux ont même été comblés de manière satisfaisante. Cependant, étant donné la situation présente, l'activité économique dans son ensemble diminue. Le marché se rétrécit ; administrativement, le pays se morcèle en républiques. Il y a beaucoup de difficultés d'approvisionnement en matières premières, surtout celles qui sont importées. Bref, les conditions économiques globales sont défavorables. Le système fiscal décourage la création d'entreprises privées ; il nuit même au maintien de celles qui existent déjà. Les impôts sont énormes; ils doivent pouvoir couvrir les dépenses budgétaires provoquécs par la guerre et par la baisse des activités économiques. Les entreprises privées ne peuvent obtenir des devises étrangères que sur le marché noir de devises, dont le cours monte chaque jour. La politique monétaire et celle du crédit sont fort peu tournées vers le développement du secteur privé. Dans l'organisation économique, représentée par le système des changes économiques, le secteur privé est faiblement représenté. Mais il convient de dire que cela est dû en bonne partie à la guerre et à la situation politique, à la nécessité d'acquérir des ressources financières pour couvrir les frais engendrés par cette guerre et à la désagrégation du système économique, tout ceci conduisant à l'épuisement total de l'économie.

Mais malgré les circonstances expressément défavorables, le secteur privé manifeste des signes de grande vitalité ; à preuve, le nombre croissant de petites entreprises et l'élargissement de leur activité économique. Même dans les conditions de guerre, les petites entreprises ont réussi à réduire quelque peu les conséquences néfastes de cette situation tragique.

Pour que les perspectives de développement des petites entreprises soient favorables, il faut que la guerre finisse et, qu'après, on trouve démocratiquement des solutions politiques pour les républiques actuelles et qu'on établisse de bonnes relations mutuelles. C'est alors que la société, pour reconstruire l'économie, pourra faire face aux terribles pertes humaines et matérielles. C'est alors qu'on verra tout l'insensé de cette horrible guerre qui a mené tous les peuples yougoslaves à la misère et, une fois de plus, à la nécessité de tout reconstruire. 
Si dans les républiques, on instaure des régimes démocratiques d'après les principes proclamés en 1989, si la reconstruction poursuit la transformation de la propriété et du marché, alors les petites entreprises joueront un grand rôle dans cette reconstruction, et plus tard, dans le développement. Mais il faut être réaliste. Les pertes et les destructions entrainées par la guerre, et les pertes causées par la désagrégation du système économique sont énormes. Il faudra beaucoup d'années de travail pour que le pays revienne au niveau de développement d'il y a à peine quelques années.

Quelle sera la réalité à venir ? On n'en sait rien. La grande chance que le pays a connue en 1989 avec le début de la réforme pour transformer le système économique est définitivement perdue. Mais si les républiques « d'après la guerre » maintiennent dans leur politique les nouveaux principes, le développement des petites entreprises deviendra un facteur essentiel de relèvement d'une économie gravement endommagée par la guerre et constituera la base d'un futur développement global.

\section{Bibliographie}

ARZEN, Sergio (1989), «The Future of SMEs in the OECD Countries », Table ronde internationale sur l'avenir de petites et moyennes entreprises. Centre européen pour la paix et le développement de l'Université pour la paix des Nations Unies, Belgrade.

Gass, James R. (1989), « The New Progress and Qualité of Life »,OECD Observer, juinjuillet.

GAss, James R. (1988), «Towards the Active Society », OECD Observer, juin-juillet.

SIPOS, Sandor (1988), «SMEs in Industrial Development : A Comparative Analysis of Hungary and South Korea », Académie des sciences de Hongrie, Budapest.

SIPOS, Sandor (1989), « The Role of SME in Transition to Market Economy in Central and East Europe », Table ronde internationale sur l'avenir de petites entreprises, Centre européen pour la paix et le développement de l'Université pour la paix des Nations Unies, Belgrade.

SEFeR, Berislav (1989), Capacités non exploitées et petite entreprise, Frères Karic, Pec-Nis.

SEFER, Berislav (1991), "Rôle des petites entreprises dans le développement de l'économie nationale. Importance des petites entreprises pour l'atténuation du problème de chômage en Yougoslavie », Centre européen pour la paix et le développement, Université pour la paix des Nations Unies, Belgrade. 